\title{
Modeling and Stability of Prey - Predator System Involving Infectious Disease in Each Population with Harvesting of the Prey
}

\author{
Nidhal Faisal Ali \\ College of Electrical and Electronic Engineering, Baghdad-Iraq. \\ E-mail: nidhal.f1980@yahoo.com.
}

\begin{abstract}
In this paper, a mathematical model consisting of the prey- predator model involving disease in both the population with harvesting of prey is proposed and analyzed. The existence, uniqueness and boundedness of the solution are discussed. The existences and the stability analysis of all possible equilibrium points are studied. Finally the effects both of the disease and harvest on the dynamical behavior the model are discussed numerically.
\end{abstract}

Keywords: eco-epidemiological model, SIS epidemics disease, prey-predator model, harvest, stability analysis.

\section{Introduction}

The effect of disease in ecological system is now becoming an important issue of research as infectious disease becomes an important factor to regulate human and animal populationsize. Anderson and May [1]; Chattopadhyay and Arino [2]; Hadeler and Freedman [3]; Venturino [4]; have been devoted to observe the dynamics of such system when prey population is infected with some transmissible diseases. Mathematics is one way to explain many of the ideas and concepts in the sciences. In the field of ecology, a lot of theoretical studies were carried out since the beginning of last century to explain the interaction between the ecological communities. One particular study describes the interaction between one population (prey) and the other (predator) living in a closed environment with the two populations ving for surviva [5].

Exploitation of biological resources as practiced in fishery, and forestry has strong impact on dynamic evolution of biological population. The over exploitation of resources may lead to extinction of species which adversely affects the ecosystem. However, reasonable and controlled harvesting is beneficial from economical and ecological point of view. The research on harvesting in predator-prey systems has been of interest to economists, ecologists and natural resource management for some time now. Very few has explicitly put a harvested parameter in a predator-prey parasite model and studied its effect on the system.
Here we study the role of harvesting in an eco-epidemiological system where the susceptible and infected prey are subjected to combined harvesting [6].

On contrast to all the above studies, in this paper a consideration is given to preypredator model involving infectious disease in both the prey and the predator species, in addition, harvest in prey species is proposed and analyzed.

In this paper a prey-predator model where the prey population infected by some infectious disease and these disease passed from a prey to predator through attacking or predation process. While the disease transmitted within the prey species by contact, between susceptible individuals and infected individuals, in additional to the external sources from the environment. It is assumed that prey-predator model involving SIS infection disease in both the prey and predator species. Further, in this model, non linear type of functional response, represented by Holloing type II as well as linear incidence rate for describing the transition of disease are used.

\section{Mathematical Model}

In this section, an eco-epidemiological model is proposed for study. The model consists of a prey, whose total population density at time $T$ is denoted by $N(T)$, interacting with predator whose total population density at time $T$ is denoted by $P(T)$. It is assumed that both the prey and the predator populations are infected by different infectious diseases. Further, the following 
assumptions are made in formulating the basic eco-epidemiological model:

1. There is an SIS epidemic disease in prey population divides the prey population into two classes namely $X(T)$ that represents the density of susceptible prey species at time $T$ and $Y(T)$ which represents the density of infected prey species at time $T$. Therefore at any $T$, we have $N(T)=X(T)+Y(T)$.

2.The disease is transmitted from a prey to predator during attacking of predator to prey, which divides the predator population into two classes namely $Z(T)$ that represents the density of susceptible predator species at time $T$ and $W(T)$ which represents the density of infected predator species at time $T$. Therefore at any $T$ we have $P(T)=Z(T)+W(T)$

3. The susceptible prey is capable of reproducing in logistic fashion with carrying capacity $k>0$, intrinsic growth $r>0$.

4. The disease transmitted within the same species (prey) by contact with an infected individual at infection rates $\lambda_{1}>0$ for the prey. In addition, there is an external source of disease causes incidence with the disease within the specific population at an external infection rates $\lambda_{2}>0$ for the prey

5. The disease disappears and infected individuals become susceptible again at the recover rates $\gamma>0$ and $\theta>0$ for prey and predator species, respectively

6. In the absence of the prey the susceptible and infected predator decay exponentially with natural death rates $d_{2}>0$ and $d_{3}>0$ respectively.

7. The disease in prey may causes mortality with a constant mortality rate represented by $d_{1}>0$.

8. The susceptible and infected predator consume the prey according to Holling type-II of functional response with maximum attack rate $\alpha_{1}>0$ and $\alpha_{2}>0$ (from susceptible prey) and $\beta_{1}>0$ and $\beta_{2}>0$ (from infected prey) respectively. However the constant $m>0$ represent the half saturation for the predator.
Considering the above basic assumptions the prey-predator model can be represented in the following system of differential equations:

$$
\begin{gathered}
\frac{d X}{d T}=r X\left(1-\frac{X+Y}{K}\right)-\left(\lambda_{1} Y+\lambda_{2}\right) X-\frac{\alpha_{1} X Z}{m+X} \\
\quad-\frac{\alpha_{2} X W}{m+X}-q_{1} X+\gamma Y \\
\frac{d Y}{d T}=\left(\lambda_{1} Y+\lambda_{2}\right) X-\frac{\beta_{1} Y Z}{m+Y}-\frac{\beta_{2} Y W}{m+Y} \\
\quad-d_{1} Y-q_{2} Y-\gamma Y \\
\frac{d Z}{d T}=-\frac{\beta_{1} Y Z}{m+Y}+\frac{e_{1} \alpha_{1} X Z}{m+X}-d_{2} Z+\theta W \\
\frac{d W}{d T}=\frac{\beta_{1} Y Z}{m+Y}+\frac{e_{2} \alpha_{2} X W}{m+X}-d_{3} W-\theta W
\end{gathered}
$$

with $X(0)>0 ; Y(0)>0 ; Z(0)>0 ; W(0)>0$ and $0<e_{i}<1 ; i=1,2$ represent the conversion rate constants and $\left(q_{1}>0\right.$ and $\left.q_{2}>0\right)$ represents the harvesting rate of susceptible and infected prey respectively. The flow of disease in system (2.1) can be described in the following block diagram.

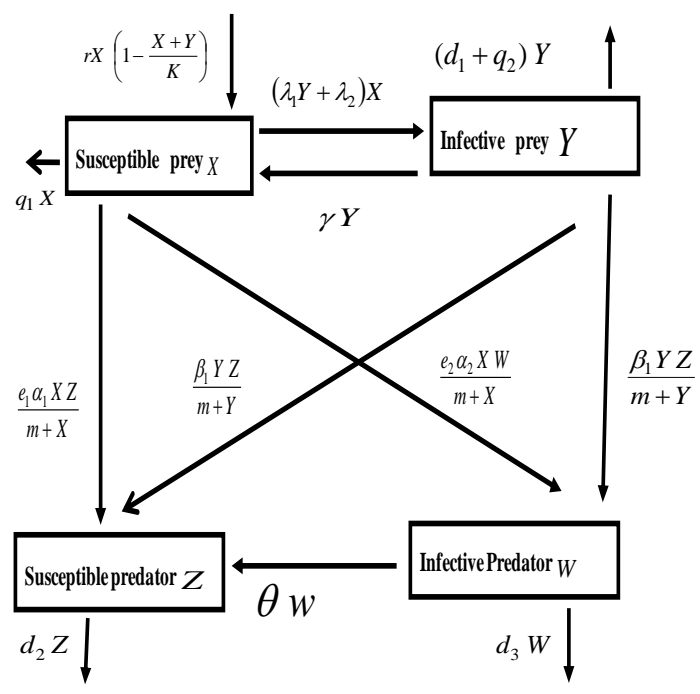

Fig.(1): Block diagram of the preypredator model given by system (1).

Cleary, system (1) included (18) parameters which make the analysis difficult. So, in order to simplify the system the number of parameters is reduced by using the following dimensionless variables

$$
t=r T, x=\frac{X}{K}, y=\frac{Y}{K}, z=\frac{Z}{K}, w=\frac{W}{K}
$$

Thus we obtain the following dimensionless form of the system (1): 


$$
\begin{aligned}
& \frac{d x}{d t}=x(1-(x+y))-\left(w_{1} y+w_{2}\right) x-\frac{w_{3} x z}{w_{5}+x}-\frac{w_{4} x w}{w_{5}+x} \\
& \quad-w_{6} x+w_{7} y=x f_{1}(x, y, z, w) \\
& \frac{d y}{d t}=\left(w_{1} y+w_{2}\right) x-\frac{w_{8} y z}{w_{5}+y}-\frac{w_{9} y w}{w_{5}+y} \\
& \quad-\left(w_{7}+w_{10}+w_{11}\right) y=y f_{2}(x, y, z, w) \\
& \frac{d z}{d t}=-\frac{w_{8} y z}{w_{5}+y}+\frac{e_{1} w_{3} x z}{w_{5}+x}-w_{12} z+w_{13} w=z f_{3}(x, y, z, w) \\
& \frac{d w}{d t}=\frac{w_{8} y z}{w_{5}+y}+\frac{e_{2} w_{4} x w}{w_{5}+x}-\left(w_{13}+w_{14}\right) w=w f_{4}(x, y, z, w)
\end{aligned}
$$

here:

$$
\begin{aligned}
& w_{1}=\frac{\lambda_{1} k}{r}>0, w_{2}=\frac{\lambda_{2}}{r}>0, w_{3}=\frac{\alpha_{1}}{r}>0 \\
& w_{4}=\frac{\alpha_{2}}{r}>0, w_{5}=\frac{m}{k}>0, w_{6}=\frac{q_{1}}{r}>0, \\
& w_{7}=\frac{\gamma}{r}>0, w_{8}=\frac{\beta_{1}}{r}>0, w_{9}=\frac{\beta_{2}}{r}>0, \\
& w_{10}=\frac{d_{1}}{r}>0, w_{11}=\frac{q_{2}}{r}>0, w_{12}=\frac{d_{2}}{r}>0, \\
& w_{13}=\frac{\theta}{r}>0, w_{14}=\frac{d_{3}}{r}
\end{aligned}
$$

represent the dimensionless parameters of the system (2). Moreover the initial condition of system (2) may be taken as any point in the region $R_{+}^{4}$. Obviously, the interaction functions in the right hand side of system (2) are continuously differentiable function on $R_{+}^{4}$, hence they are Lipschitizian. Therefore the solution of system (2) exists and is unique. Further, all the solutions of system (2) with $\geq 0$ initial condition are uniformly bounded as shown in the following theorem.

\section{Theorem (1):}

All the solutions of the system (2) are uniformly bounded.

\section{Proof:}

Let $x(t), y(t), z(t), w(t)$ be any solution of the system (2). Define the function $M(t)=x(t)+y(t)+z(t)+w(t), \quad$ then the time derivative of $M(t)$ along the solution of the system (2), gives

$$
\frac{d M}{d t}=\frac{d x}{d t}+\frac{d y}{d t}+\frac{d z}{d t}+\frac{d w}{d t}
$$

$$
\begin{gathered}
\frac{d M}{d t}=x(1-(x+y))-\left(w_{1} y+w_{2}\right) x-\frac{w_{3} x z}{w_{5}+x}-\frac{w_{4} x w}{w_{5}+x} \\
-w_{6} x+w_{7} y\left(w_{1} y+w_{2}\right) x-\frac{w_{8} y z}{w_{5}+y} \\
-\frac{w_{9} y w}{w_{5}+y}-\left(w_{7}+w_{10}+w_{11}\right) y-\frac{w_{8} y z}{w_{5}+y} \\
+\frac{e_{1} w_{3} x z}{w_{5}+x}-w_{12} z+w_{13} w+\frac{w_{8} y z}{w_{5}+y} \\
+\frac{e_{2} w_{4} x w}{w_{5}+x}-\left(w_{13}+w_{14}\right) w
\end{gathered}
$$

$$
\begin{gathered}
\frac{d M}{d t} \leq x-x^{2}-x y-\left(1-e_{1}\right) \frac{w_{3} x z}{w_{5}+x} \\
-\left(1-e_{2}\right) \frac{w_{4} x w}{w_{5}+x}-w_{6} x-\frac{w_{9} y w}{w_{5}+y} \\
-\left(w_{10}+w_{11}\right) y-\frac{w_{8} y z}{w_{5}+y}-w_{12} z \\
-w_{14} w
\end{gathered}
$$

Then according to the theory of differential inequality we have sup $x(t) \leq 1, \forall t \geq 0$

$$
\frac{d M}{d t} \leq 1-n M
$$

where $n=\min \left\{w_{6}, w_{10}+w_{11}, w_{12}, w_{14}\right\}$.

Now, by using Gromwell lemma, it obtains that: $0<M(t) \leq M(0) e^{-n t}+\frac{1}{n}\left(1-e^{-n t}\right) \quad$ which yields $\lim _{t \rightarrow \infty} M(t) \leq \frac{1}{n}$ that is independent of the initial conditions. Thus the proof is complete.

\section{Existence of equilibrium points:}

It is observed that, system (2) has at most three biologically feasible equilibrium points, namely $E_{i}=(x, y, z, w), i=0,1,2$. The existence conditions for each of these equilibrium points are discussed in the following:

1. The vanishing equilibrium point

$E_{0}=(0,0,0,0)$ always exists.

2 . The predator free equilibrium point $E_{1}=(\hat{x}, \hat{y}, 0,0)$

where

$\hat{y}=\frac{w_{2} \hat{x}}{\left(w_{7}+w_{10}+w_{11}\right)-w_{1} \hat{x}} ;\left(w_{7}+w_{10}+w_{11}\right) \neq w_{1} \hat{x}$ 
while $\hat{x}$ represents a positive root of the following second order polynomial equation

$$
B_{1} x^{2}+B_{2} x+B_{3}=0
$$

where:

$$
\begin{aligned}
B_{1}= & w_{1}>0 \\
B_{2}= & w_{1}\left(w_{2}+w_{6}\right) \\
& -\left[w_{7}+w_{10}+w_{11}+w_{1}+w_{2}\left(1+w_{1}\right)\right] ; \\
B_{3}= & w_{2} w_{7}+w_{7}+w_{10}+w_{11} \\
& -\left[\left(w_{7}+w_{10}+w_{11}\right)\left(w_{2}+w_{6}\right)\right]
\end{aligned}
$$

Consequently, straightforward computation shows that $E_{1}$ exists uniquely in the interior of the first quadrant of $x y-$ plane if and only if the following condition are hold.

$$
B_{3}<0
$$

\section{The positive equilibrium point}

$$
E_{2}=\left(x^{*}, y^{*}, z^{*}, w^{*}\right)
$$

The positive equilibrium point $E_{2}$ exists in the Int. $R_{+}^{4}$ if and only if there is a positive solution of the following set of algebraic equations

$$
\begin{aligned}
f_{1}(x, y, z, w) & =1-(x+y)-\left(w_{1} y+w_{2}\right)-\frac{w_{3} z}{w_{5}+x} \\
- & \frac{w_{4} w}{w_{5}+x}-w_{6}+w_{7} \frac{y}{x}=0
\end{aligned}
$$

$$
\begin{gathered}
f_{2}(x, y, z, w)=\left(w_{1}+\frac{w_{2}}{y}\right) x-\frac{w_{8} z}{w_{5}+y}-\frac{w_{9} w}{w_{5}+y} \\
-\left(w_{7}+w_{10}+w_{11}\right)=0
\end{gathered}
$$

$$
f_{3}(x, y, z, w)=-\frac{w_{8} y}{w_{5}+y}+\frac{e_{1} w_{3} x}{w_{5}+x}-w_{12}+w_{13} \frac{w}{z}=0
$$

$$
f_{4}(x, y, z, w)=\frac{w_{8} y z}{w\left(w_{5}+y\right)}+\frac{e_{2} w_{4} x}{w_{5}+x}-\left(w_{13}+w_{14}\right)=0
$$

By solving (6b) and (6d), we obtain that

$$
z(x, y)=\frac{B^{2} C\left[\left(w_{13}+w_{14}\right) A-e_{2} w_{4} x\right]}{w_{8} y D} ; D \neq 0
$$

$$
w(x, y)=\frac{A B C}{D} ; D \neq 0
$$

Where

$$
\begin{aligned}
& A=w_{5}+x, B=w_{5}+y, C=y\left(w_{1} x-a\right)+w_{2} x, \\
& D=B\left[\left(w_{13}+w_{14}\right) A-e_{2} w_{4} x\right]+w_{9} y A
\end{aligned}
$$

Then by using (6e) and (6f) in (6a) and (6c) yield the following two isoclines.

$$
\begin{gathered}
g_{1}(x, y)=y\left[1-(x+y)-\left(w_{1} y+w_{2}\right)-\frac{w_{4} B C}{D}-w_{6}+\frac{w_{7} y}{x}\right] \\
-\frac{w_{3} B^{2} C\left[\left(w_{13}+w_{14}\right) A-e_{2} w_{4}\right]}{w_{8} A D}=0
\end{gathered}
$$

$$
g_{2}(x, y)=y\left[\frac{w_{8} w_{13} A}{B\left[\left(w_{13}+w_{14}\right) A-e_{2} w_{4}\right]}-\frac{w_{8}}{B}\right]+\frac{e_{1} w_{3} x}{A}
$$

Now from $(6 \mathrm{~g})$ we notice that, when $\mathrm{y} \rightarrow 0$, then $x \rightarrow x_{1}$ so that:

$$
x_{1}=\frac{w_{5}\left(w_{13}+w_{14}\right)}{e_{2} w_{4}-\left(w_{13}+w_{14}\right)}
$$

Obviously, $x_{1}>0$ provided that:

$e_{2} w_{4}>\left(w_{13}+w_{14}\right)$

Further, from (6h) we notice that, when $y \rightarrow 0$ then, $\quad x \rightarrow x_{2}$ so that:

$$
x_{2}=\frac{w_{12} w_{5}}{e_{1} w_{3}-w_{12}}
$$

Obviously, $x_{2}>0$ provide that:

$e_{1} w_{3}>w_{12}$

Now, we assume that $x_{2}>x_{1}$. Then from Eq. (6g) we have

$$
\frac{d x}{d y}=-\left(\frac{\partial g_{1}}{\partial y}\right) /\left(\frac{\partial g_{1}}{\partial x}\right) \text {. So, } \frac{d x}{d y}>0 \text { if one set }
$$
of the following sets of conditions holds.

$$
\frac{\partial g_{1}}{\partial x}>0, \frac{\partial g_{1}}{\partial y}<0 \text { OR } \frac{\partial g_{1}}{\partial x}<0, \frac{\partial g_{1}}{\partial y}>0
$$

Further, from (6h) we notice

that $\frac{d x}{d y}=-\left(\frac{\partial g_{2}}{\partial y}\right) /\left(\frac{\partial g_{2}}{\partial x}\right)$. So, $\frac{d x}{d y}<0$ if one set of the following sets of conditions holds. 


$$
\frac{\partial g_{2}}{\partial x}>0, \frac{\partial g_{2}}{\partial y}>0 \text { OR } \frac{\partial g_{2}}{\partial x}<0, \frac{\partial g_{2}}{\partial y}<0
$$

Then the two isoclines (6g) and (6h) intersect at a unique positive point $\left(x^{*}, y^{*}\right)$ in the Int. $R_{+}^{2}$ of $x y$-plane. Substituting the value of $x^{*}$ and $y^{*}$ in Eq. (6e) and (6f) yield that $z\left(x^{*}, y^{*}\right)=z^{*}$ and $w\left(x^{*}, y^{*}\right)=w^{*}$ which are positive if and only if the following condition holds.

$$
\frac{\left(w_{7}+w_{10}+w_{11}\right)}{w_{1}}<x^{*}<\frac{A\left(w_{13}+w_{14}\right)}{e_{2} w_{4}}
$$

Accordingly, the positive equilibrium point $E_{2}$ exists uniquely in the Int. $R_{+}^{4}$ if in addition to the conditions (8), (10), (13) and $x_{2}>x_{1}$; the isoclinic $g_{1}(x, y)=0$ intersect the $\mathrm{x}$-axis at the positive value namely $x_{1}$ and the isoclinic $g_{2}(x, y)=0$ intersect the $\mathrm{x}$-axis at the positive value namely $x_{2}$.

\section{The local Stability Analysis:}

In this section, the local stability analyses of system (2) around each of the above equilibrium points of system (2) are studied with the help of Linearization method. Note that the symbols $\lambda_{i x}, \lambda_{i y}, \lambda_{i z}$ and $\lambda_{i w}$ denote to the eigenvalues of the Jacobian matrix $J\left(E_{i}\right) ; i=0,1,2$ that describe the dynamics in the $x$ - direction $y$-direction, $z$-direction and $w$-direction, respectively.

The Jacobian matrix of system (2) at $E_{0}$ can be written as:

$$
J\left(E_{0}\right)=\left[\psi_{i j}\right]_{4 \times 4} ; i, j=1,2,3,4
$$

\section{Where :}

$$
\begin{aligned}
& \psi_{11}=1-\left(w_{2}+w_{6}\right) ; \psi_{12}=w_{7} ; \psi_{13}=\psi_{14}=0 ; \\
& \psi_{21}=w_{2}>0 ; \psi_{22}=-\left(w_{7}+w_{10}+w_{11}\right)<0 ; \\
& \psi_{23}=\psi_{24}=0 ; \psi_{31}=\psi_{32}=0 ; \psi_{33}=-w_{12}<0 ; \\
& \psi_{34}=w_{13}>0 ; \psi_{41}=\psi_{42}=\psi_{43}=0 ; \\
& \psi_{44}=-\left(w_{13}+w_{14}\right)<0
\end{aligned}
$$

Clearly the characteristic equation of $J_{0}$ can be written

$$
\begin{aligned}
& {\left[\lambda_{0}^{2}-\left(\psi_{11}+\psi_{22}\right) \lambda_{0}+\psi_{11} \psi_{22}-\psi_{12} \psi_{21}\right]} \\
& {\left[\lambda_{0}^{2}-\left(\psi_{33}+\psi_{44}\right) \lambda_{0}+\psi_{33} \psi_{44}\right]=0}
\end{aligned}
$$

Therefore, the eigenvalus of $J_{0}$ satisfy the following relations:

$$
\begin{aligned}
& \lambda_{0 x}+\lambda_{0 y}=\psi_{11}+\psi_{22} \\
& \lambda_{0 x} \cdot \lambda_{0 y}=\psi_{11} \psi_{22}-\psi_{12} \psi_{2121} \\
& \lambda_{0 z}+\lambda_{0 w}=\psi_{33}+\psi_{44} \\
& \lambda_{0 z} \cdot \lambda_{0 w}=\psi_{33} \psi_{44}
\end{aligned}
$$

Clearly according to the following condition all the eigenvalues have negative real parts.

$$
\operatorname{Max}\left\{\begin{aligned}
w_{7} & \left.+w_{10}+w_{11}, \frac{w_{7}+w_{10}+w_{11}+w_{2}+w_{6}}{w_{7}+w_{10}+w_{11}}\right\} \\
& <\left(w_{2}+w_{6}\right)
\end{aligned}\right.
$$

Hence the equilibrium point $E_{0}$ is locally asymptotically stable in $R_{+}^{4}$.

The Jacobian matrix of system (2) at the predator free equilibrium point $E_{1}=(\hat{x}, \hat{y}, 0,0)$ can be written as:

$$
J\left(E_{1}\right)=\left[e_{i j}\right]_{4 \times 4 ; i, j=1,2,3,4}
$$

where

$e_{11}=1-\left(w_{2}+w_{6}+2 \hat{x}+\left(1+w_{1}\right) \hat{y}\right) ;$

$e_{12}=w_{7}-\left(1+w_{1}\right) \hat{x} ; e_{13}=\frac{-w_{3} \hat{x}}{w_{5}+\hat{x}}<0 ;$

$e_{14}=-\frac{-w_{4} \hat{x}}{w_{5}+\hat{x}}<0 ; e_{21}=w_{1} \hat{y}+w_{2}>0 ;$

$e_{22}=w_{1} \hat{x}-\left(w_{7}+w_{10}+w_{11}\right)$

$e_{23}=\frac{-w_{8} \hat{y}}{w_{5}+\hat{y}}<0 ; e_{24}=-\frac{-w_{9} \hat{y}}{w_{5}+\hat{y}}<0 ;$

$e_{31}=e_{32}=0 ; e_{33}=\frac{e_{1} w_{3} \hat{x}}{w_{5}+\hat{x}}-\left(\frac{w_{8} \hat{y}}{w_{5}+\hat{y}}+w_{12}\right) ;$

$e_{34}=w_{13}>0 ; e_{41}=e_{42}=0 ; e_{43}=\frac{w_{8} \hat{y}}{w_{5}+\hat{y}}>0$;

$e_{44}=\frac{e_{2} w_{4} \hat{x}}{w_{5}+\hat{y}}-\left(w_{13}+w_{14}\right)$

Clearly the characteristic equation of $J_{1}$ can be written

$$
\begin{aligned}
& {\left[\lambda_{1}^{2}-\left(e_{11}+e_{22}\right) \lambda_{1}+e_{11} e_{22}-e_{12} e_{21}\right]} \\
& \quad\left[\lambda_{1}^{2}-\left(e_{33}+e_{44}\right) \lambda_{1}+e_{33} e_{44}-e_{34} e_{43}\right]=0
\end{aligned}
$$

Therefore, the eigenvalus of $J_{1}$ satisfy the following relations: 


$$
\begin{aligned}
& \lambda_{1 x}+\lambda_{1 y}=e_{11}+e_{22} \\
& \lambda_{1 x} \cdot \lambda_{1 y}=e_{11} e_{22}-e_{12} e_{21} \\
& \lambda_{1 z}+\lambda_{1 w}=e_{33}+e_{44} \\
& \lambda_{1 z} \cdot \lambda_{1 w}=e_{33} e_{44}-e_{34} e_{43}
\end{aligned}
$$

Clearly according to the following conditions all the eigenvalues have negative real parts.

$$
\begin{aligned}
& e_{11}<0 \text { and } e_{22}<0 \\
& e_{12}<0 \\
& e_{33}<0 \text { and } e_{44}<0 \\
& e_{34} e_{43}<e_{33} e_{44}
\end{aligned}
$$

Hence the equilibrium point $E_{1}$ is locally asymptotically stable in $R_{+}^{4}$

In the following theorem, the local stability conditions of the positive equilibrium point $E_{2}$ are established.

\section{Theorem (2) :}

Assume that $E_{2}=\left(x^{*}, y^{*}, z^{*} w^{*}\right)$ exists in the Int $R_{+}^{4}$ and the following condition are satisfied

$$
\begin{aligned}
& \operatorname{Max} .\left\{N_{3}\right\}<x^{*}<\operatorname{Min} .\left\{N_{4}, N_{5}, N_{6}\right\} \\
& \frac{1}{N_{1}^{2}}<\operatorname{Min} .\left\{N_{7}, N_{10}\right\} \\
& \frac{1}{N_{2}^{2}}<\operatorname{Min} .\left\{N_{8}, N_{9}\right\}
\end{aligned}
$$

here $N_{i}, i=1, \ldots ., 10$ are given in proof . Then the positive equilibrium point $E_{2}$ is locally asymptotically stable in the Int $R_{+}^{4}$

\section{Proof:}

The Jacobian matrix of system (2) at the positive equilibrium point $E_{2}$ can be written:

$$
J\left(E_{2}\right)=\left\lfloor r_{i j}\right\rfloor_{4 \times 4} ; i, j=1,2,3,4
$$

where:

$$
r_{11}=1-\left[\begin{array}{r}
w_{2}+w_{6}+2 x^{*}+\left(1+w_{1}\right) y^{*} \\
+\frac{w_{5}\left(w_{3} z^{*}+w_{4} w^{*}\right)}{N_{1}^{2}}
\end{array}\right]
$$

$$
\begin{aligned}
& r_{12}=\left[w_{7}-\left(1+w_{1}\right) x^{*}\right] r_{13}=-\frac{w_{3} x^{*}}{N_{1}}<0 ; \\
& r_{14}=-\frac{w_{4} x^{*}}{N_{1}}<0 ; r_{21}=w_{1} y^{*}+w_{2}>0 ;
\end{aligned}
$$$$
r_{22}=w_{1} x^{*}-\left(w_{7}+w_{10}+w_{11}+\frac{w_{5}\left(w_{8} z^{*}+w^{*}\right)}{N_{2}^{2}}\right) ;
$$$$
r_{23}=-\frac{w_{8} y^{*}}{N_{2}}<0 ; r_{24}=-\frac{w_{9} y^{*}}{N_{2}}<0 ; r_{31}=\frac{e_{1} w_{3} w_{5} z^{*}}{N_{1}^{2}}>0 \text {; }
$$$$
r_{32}=-\frac{w_{5} w_{8} z^{*}}{N_{2}^{2}}<0
$$$$
r_{33}=\frac{e_{1} w_{3} x^{*}}{N_{1}}-\left(\frac{w_{9} y^{*}}{N_{2}}+w_{12}\right) \text {; }
$$$$
r_{34}=w_{13}>0 ; r_{41}=\frac{e_{2} w_{4} w_{5} w^{*}}{N_{1}^{2}}>0 ; r_{42}=\frac{w_{5} w_{8} z^{*}}{N_{2}^{2}}>0 ;
$$$$
r_{43}=\frac{w_{8} y^{*}}{N_{2}}>0 ; r_{44}=\frac{e_{2} w_{4} x^{*}}{N_{1}}-\left(w_{13}+w_{14}\right)
$$

with

$$
\begin{aligned}
& N_{1}=w_{5}+x^{*}, \\
& N_{2}=w_{5}+y^{*} \text {, } \\
& N_{3}=\frac{1}{2}\left(\begin{array}{c}
1-\left[w_{2}+w_{6}+\left(1+w_{1}\right) y^{*}\right] \\
-\frac{w_{5}\left(w_{3} z^{*}+w_{4} w^{*}\right)}{N_{1}^{2}}
\end{array}\right), \\
& N_{4}=\frac{1}{w_{1}}\left(\frac{w_{5}\left(w_{3} z^{*}+w^{*}\right)}{N_{2}^{2}}\right) \text {, } \\
& N_{5}=\frac{N_{1}}{e_{1} w_{3}}\left(w_{12}+\frac{w_{8} y^{*}}{N_{2}}\right) \text {, } \\
& N_{6}=\frac{N_{1}}{e_{2} w_{4}}\left(w_{13}+w_{14}\right) \text {, } \\
& N_{7}=\left(\frac{1-\left[2 w_{2}+w_{6}+2 x^{*}+\left(1+2 w_{1}\right) y^{*}\right]}{w_{5}\left(e_{1} w_{3} z^{*}+e_{2} w_{4} w^{*}+N_{1}\left(w_{3} z^{*}+w^{*}\right)\right.}\right), \\
& N_{8}=\left(\frac{w_{1} x^{*}-\left(w_{7}+w_{10}+w_{11}\right)-\left|w_{7}-x^{*}\left(1+w_{1}\right)\right|}{w_{5} z^{*}\left(3 w_{8}+1\right)}\right) \text {, } \\
& N_{9}=\left(\frac{1}{3 w_{8} y^{*}}\left[\frac{\left(e_{1}-1\right) w_{3} x^{*}-N_{1} w_{12}}{N_{1}}\right]\right)^{2}, \\
& N_{10}=\left(\frac{1}{w_{4} x^{*}}\left[\frac{e_{2} w_{4} x^{*}-w_{9} y^{*}-}{N_{2}}-\left(2 w_{13}+w_{14}\right)\right]\right)^{2}
\end{aligned}
$$


Now according to the conditions (29) and (30); all the values of $r_{11}, r_{22}, r_{33}$ and $r_{44}$ are negative values. So according to Gersgorin theorem the proof is follows if and only if $\left|r_{i i}\right|>\sum_{i=1}^{4}\left|r_{i j}\right|$, and $i \neq j$

then all the eigenvalues of $J\left(E_{2}\right)$ exists in the region

$$
\xi=\cup\left\{U^{*} \in C:\left|U^{*}-r_{i i}\right|<\sum_{\substack{i=1 \\ i \neq j}}^{4}\left|r_{i j}\right|\right\}
$$

Clearly, its easy to show that under the given conditions (32) all the eigenvalues of $J\left(E_{2}\right)$ exists in the left half plan and hence $E_{2}$ is

Locally asymptotically stable.

\section{Numerical analysis of system (2)}

In this section the dynamical behavior of system (2) is studied numerically by using Runge Kutta method along with predector corrector method. Note that, we use turbo $\mathrm{C}++$ in programming and matlab. The system (2) is studied numerically for different sets of parameters and different sets of initial points. The objectives of this study are: first investigate the effect of varying the value of each parameter on the dynamical behavior of system (2) and second confirm our obtained analytical results. It is observed that, for the following set of hypothetical parameters:

$$
\begin{aligned}
& w_{1}=0.6, w_{2}=0.5, w_{3}=0.75, \\
& w_{4}=0.5, w_{5}=0.5, \quad w_{6}=0.1, w_{7}=0.05, \\
& w_{8}=0.2, w_{9}=0.25, w_{10}=0.05 \\
& w_{11}=0.1, w_{12}=0.001, \quad w_{13}=0.05 \\
& w_{14}=0.01, \quad e_{1}=0.7, \quad e_{2}=0.4
\end{aligned}
$$

Note that, in Fig. (2), we will use that ( ) to describe the trajectory of system (2) that started at $(0.0,0.8,0.7,0.6,0.5)$ and $(\ldots .$.$) to$ describe the trajectory of system (2) that started at $(0.5,0.3,0.3,0.2)$.
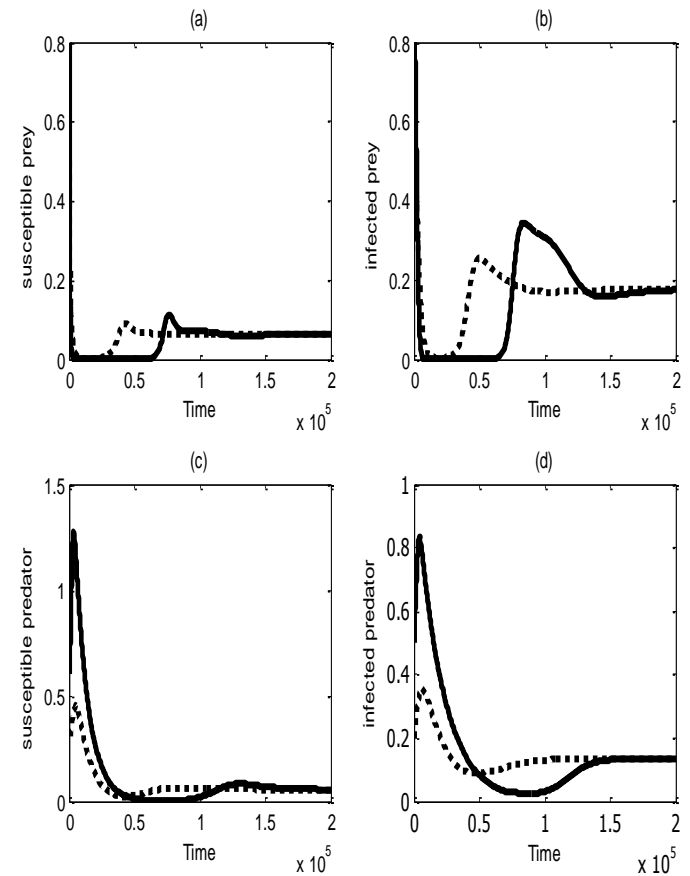

Fig.(2): Time series of the solution of system (2)

(a) trajectories of $x$ as a function of time, (b) trajectories of $y$ as a function of time,

(c) trajectories of $z$ as a function of time,

(d) trajectories of $w$ as a function of time.

Clearly, Fig. (2) shows that the solution of system (2) approaches asymptotically to the positive equilibrium point $E^{*}=(0.4,0.1,0.2,0.3)$ starting from two different initial points and this is confirming our obtained analytical results.

Now in order to discuss the effect of the parameters values of system (2) on the dynamical behavior of the system, the system is solved numerically for the data given in Eq. (33) with varying one parameter each time. It is observed that varying the parameters values $w_{i} ; i=1,2,4,5,7,9,10,12,13,14$ and $e_{i} ; i=1$ do not have any effect on the dynamical behavior of system (2) and the system still approaches to positive equilibrium point. For the parameters values given in Eq. (33) with varying $w_{3}$ in the range $w_{3} \leq 0.45$, the solution of the system (2) approaches to $E_{2}=(\hat{x}, \hat{y}, 0,0)$ in the interior of positive quadrant of $x y$-plane, as shown in Fig.(3), however for $w_{3} \geq 0.46$ it is observed that the solution of system (2) approaches to positive equilibrium point. 


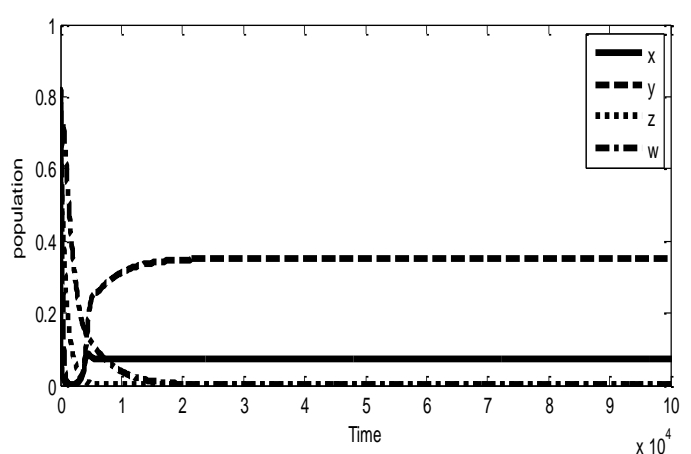

Fig. (4): Time series of the solution of system

(2) for the data in given by Eq.(33) with $w_{3}=0.45$, which approaches asymptotically to $(0.57,0.61,0,0)$ the interior of positive quadrant of $x y$-plane.

For the parameters values given in Eq. (33) with varying $w_{6}$ in the range $w_{6} \leq 0.3$ the solution approaches to the solution of system (2) approaches to positive equilibrium point , while for $0.4 \leq w_{6} \leq 0.6$ the solution of the system (2) approaches to $E_{2}=(\hat{x}, \hat{y}, 0,0)$ in the interior of positive quadrant of $x y$-plane, finally for $0.7 \leq w_{6}$ system (2) approaches to the vanishing equilibrium point $E_{0}=(0,0,0,0)$ as shown in Fig.(5) .

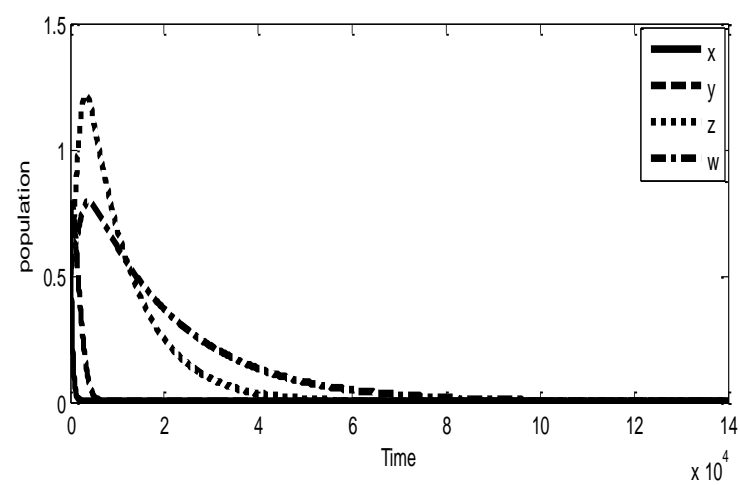

Fig.(5): Time series of the solution of system

(2) for the data given by Eq. (33) with $w_{3}=0.7$, which approaches asymptotically

to the vanishing equilibrium point.

On the other hand varying the parameter $w_{8}$ keeping the rest of parameters values as in Eq. (33), it observed that for $w_{8} \leq 0.5$ the solution of system (2) approaches asymptotically to positive equilibrium point, while for $0.6 \leq w_{8}$ approaches to $E_{2}=(\hat{x}, \hat{y}, 0,0)$ in the interior of positive quadrant of $x y$-plane. For the parameters values given in Eq. (33) with varying $w_{11}$ in the range $w_{11} \leq 0.2$ the solution approaches to positive equilibrium point, while for $0.3 \leq w_{11}$ the solution of the system (2) approaches to $E_{2}=(\hat{x}, \hat{y}, 0,0)$ in the interior of positive quadrant of $x y$-plane, Finally, For the parameters values given in Eq. (33) with varying $e_{1}$ in the range $e_{1} \leq 0.4$, the solution of the system (2) approaches to $E_{2}=(\hat{x}, \hat{y}, 0,0)$ in the interior of positive quadrant of $x y-$ plane, as shown in Fig.(3) ,however for $w_{3} \geq 0.5$ it is observed that the solution of system (2) approaches to positive equilibrium point.

\section{Conclusions and Discussion}

In this paper, an eco-epidemiological model has been proposed and analyzed. In order to study the effect of infection diseases and harvesting on the dynamical behavior of the prey-predator system, the dynamical behavior of system (2) has been investigated locally. In addition to assumed that the prey population is harvested and the prey population infected by some infectious disease and these disease passed from a prey to predator through attacking or predation process. While the disease transmitted within the prey species by contact, between susceptible individuals and infected individuals, in additional to the external sources from the environment. It is assumed that prey- predator model involving SIS infection disease in both the prey and predator species. Further, in this model, non linear type of functional response, represented by Holloing type II as well as linear incidence rate for describing the transition of disease are used. The model included four non-linear autonomous differential equations that describe the dynamics of four different population namely susceptible prey $x$, infected prey $y$, susceptible predator $z$, infected predator $w$.

To understand the effect of varying each parameter on the global dynamics of system (2) and to confirm our obtained analytical results, system (2) has been solved numerically and the following results are obtained

1. The system (2) dose not have periodic dynamic. 
2. For the set of hypothetical parameters values given Eq. (33), system (2) approaches asymptotically to a globally asymptoticallystable point $E_{2}=\left(x^{*}, y^{*}, z^{*}, w^{*}\right)$.

3. It is observed that varying the parameters values $w_{i} ; i=1,2,4,5,7,9,10,12,13,14$ and/ $e_{i} ; i=1$ keeping other parameters as given by Eq. (33) do not have any effect on the dynamical behavior of system (2) and the system still approaches to a positive equilibrium point.

As the susceptible prey's maximum attack rate by susceptible predator $w_{3}$ decreases slightly keeping the rest of parameters as in Eq. (33), the system has asymptotically stable equilibrium point $L_{2}=(\hat{x}, \hat{y}, 0,0)$.

However increasing the parameter $w_{3}$ system (2) still has asymptotically stable positive point in the Int. $R_{+}^{4}$. It is observed that the conversion rate from susceptible prey to susceptible predator parameter $e_{1}$ respectively, have the same effect as $w_{3}$.

4. As the harvesting rate of susceptible prey $w_{6}$ decreases keeping the rest of parameters as in Eq. (33), syatem (2) still has asymptotically stable positive point in the Int. $R_{+}^{4}$. However, increasing $w_{6}$ causes extinction in the both predator species and the solution of system (2) approaches to the equilibrium point

$E_{2}=(\hat{x}, \hat{y}, 0,0)$. While, increasing $w_{6}$ causes extinction in the both predator and prey species and the solution of system (2) approaches to the equilibrium point $E_{2}=(0,0,0,0)$.

As the maximum attack rate of the infected prey by susceptible predator $w_{8}$ decreases keeping the rest of parameters as in Eq. (33), syatem (2) still has asymptotically stable positive point in the Int. $R_{+}^{4}$. However, increasing $w_{6}$ causes extinction in the both predator species and the solution of system (2) approaches to the equilibrium point $E_{2}=(\hat{x}, \hat{y}, 0,0)$. It is observed that the harvesting rate of infected prey $w_{11}$ as the same effect as $w_{8}$.

\section{References}

[1] Anderson R.M., May R. M., "The invasion. Persistence, and spread of Infectious diseases within animal and plant communities"; Philos, Trans. R, Soc. Lond. Ser. B, $314-570,1986$.

[2] Chattopadhyay J., Arino, O., "A Predator prey model with disease in the Nonlinear Anal"; 36,747-766, 1999.

[3] Hadeler K.P., Freedman H.1., "predator prey populations with parasitic infection"; J. Math. BIO, 27, 1609-631, 1989.

[4] Venturion E., "Epidemics in predator prey models: disease in the prey"; In: Arin O; Axelrod D., Kimmel M. and Langlais M. (Eds.), Mathematical population Dynamics: Analysis of Heterogeneity, Theory of Epidemics. Wuerz puplishing Winnipeg. Canada, 381 - 393, 1995.

[5] Wuhaib Y, Abu Hasan S.A; "A predatorinfected prey model with harvesting of infected prey"; School of Mathematical Sciences, Universiti Sains Malaysia, 11800 Penang Malays, 2013.

[6] Kul Bhushan Agnihotri; "The dynamics of disease transmission in a prey predator system with harvesting of prey"; International Journal of Advanced Research in Computer Engineering \& Technology, 1, April 2012.

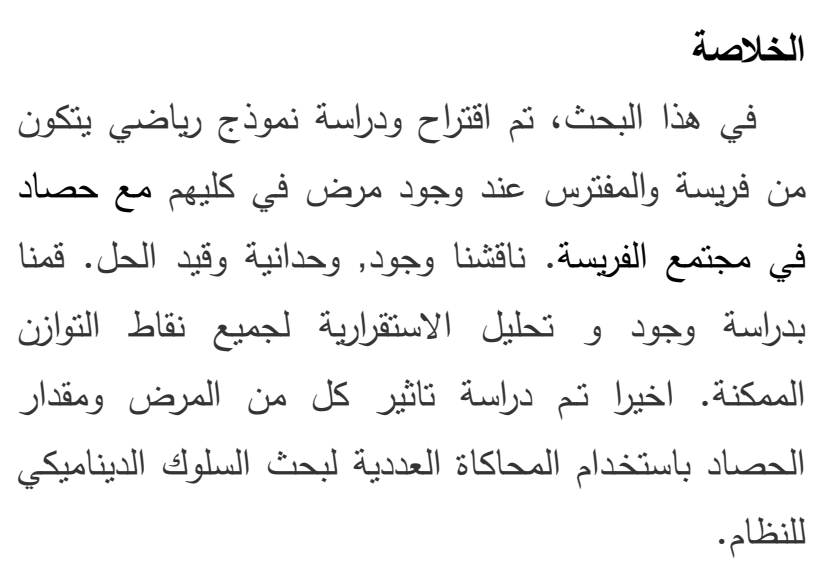

